\section{Estilos de Gestión Educativa y Clima Institucional en IE públicas en el distrito de Carmen de la Legua}

\author{
Styles of educational Management and Institutional Climate \\ in public IE in the district of Carmen de la Legua
}

\begin{abstract}
RESUMEN
Este artículo presenta las conclusiones más relevantes de la investigación "Relación que existe entre el estilo de gestión educativa de los directivos y el Clima Institucional de IE Públicas en el distrito de Carmen de la Legua”, este estudio consiste en el análisis de las variables Estilo de Gestión Educativa y Clima Institucional. La investigación metodológicamente es de tipo Transaccional o Transversal: Correlacional. La finalidad del presente trabajo de investigación fue conocer el tipo de gestión educativa y su implicancia en el Clima Institucional, la cual se conoció a través del estadístico CHI cuadrado. Existe una asociación significativa con un p _ Valor de 0,000 al 95\% así de esta manera se rechaza la hipótesis nula y se admite la hipótesis alterna general, la cual nos dice que hay existe una correlación de gran importancia en las variables estilo de gestión educativa y clima institucional de IE Públicas en el distrito de Carmen de la Legua.
\end{abstract}

Palabras claves: Clima Institucional; Gestión Educativa; Estilo de gestión.

\section{ABSTRACT}

This article presents the most relevant conclusions of the research "Relationship between the style of educational management of managers and the institutional climate of public educational institutions in the district of Carmen de la Legua, this study is based on the analysis of the relationships of the variables style Educational Management and institutional Climate. The research methodological is Transactional type or transverse: Correlational. The purpose of this research is to know the type of Educational Management and its implication in the institutional climate. Which became known through statistical square $\mathrm{CHI}$ existence a significant association with a $\mathrm{p}_{\text {- }}$ value of 0.000 to $95 \%$ therefore the null hypothesis is rejected and generally accepted alternative which tells us that there is significant relationship between Management Style Educational Managers and Institutional Climate of Educational institutions of governance in the district of Carmen de la Legua. Keywords: Institutional Climate; Education Management; Management Style.

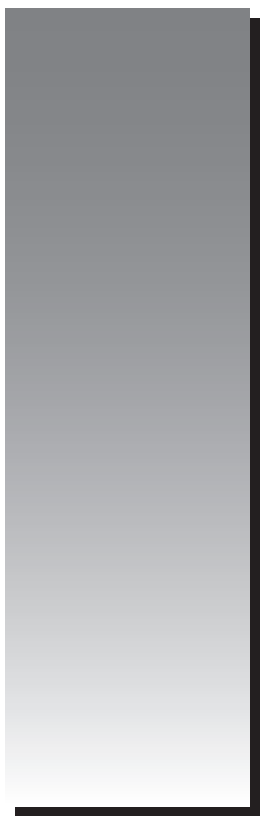

Rocio del Pilar Fasanando Guabloche ${ }^{1}$ sagit_16rpfg@hotmail.com

Universidad Nacional Mayor de San Marcos

1 Estudiante de Doctorado en Ciencias Administrativas, Magíster en Administración, Docente auxiliar en la facultad de Ciencias Administrativas de la Universidad Nacional Mayor de San Marcos.

(C) Los autores. Este artículo es publicado por Gestión en el Tercer Milenio de la Facultad de Ciencias Administrativas de la Universidad Nacional Mayor de San Marcos. Este es un artículo de acceso abierto, distribuido bajo los términos de la licencia Creative Commons Atribucion - No Comercia_Compartir Igual 4.0 Internacional. (http://creativecommons.org/licenses/by-nc-sa/4.0/) que permite el uso no comercial, distribución y reproducción en cualquier medio, siempre que la obra original sea debidamente citada. 


\section{INTRODUCCIÓN}

El estilo de gestión que aplican los directivos en las IE Públicas es de vital importancia ya que repercute en el Clima Institucional. Un buen estilo de gestión garantiza que la educación sea de calidad. La Institución Educativa de calidad es aquella entidad promotora del progreso de los estudiantes en un amplio matiz de logros tanto intelectual, moral, emocional y social. La educación de calidad es aquella que ayuda al estudiante a satisfacer plenamente sus necesidades y a desarrollar sus habilidades personales.

Teniendo en cuenta el valioso significado e importancia del estilo de gestión educativa se ha realizado el presente estudio, cuyo propósito primordial es analizar cada una de las variables, para determinar la relación que hay entre ellas, como influyen en el Clima institucional y sus repercusiones.

La presente investigación se realiza porque actualmente en las IE públicas de educación secundaria del distrito de Carmen de La Legua, se evidencia una serie de situaciones en la cual el estilo de gestión que realizan los directivos, unos con acierto y otros con desacierto, podría repercutir en el Clima institucional de cada institución.

\section{Formulación del Problema}

\section{General}

¿Qué correlación existe entre los estilos de gestión educativa y el Clima Institucional en IE Públicas del distrito de Carmen de La Legua?

\section{Secundarios}

- ¿Qué relación hay entre el estilo autoritario y el Clima Institucional de IE Públicas del distrito de Carmen de La Legua?

- ¿Qué relación hay entre el estilo democrático y el Clima Institucional de IE Públicas del distrito de Carmen de La Legua?

- ¿Qué relación hay entre el estilo leissez Faire y el Clima Institucional de IE Públicas del distrito de Carmen de la Legua?

\section{Objetivos}

\section{General}

Establecer la correlación que hay entre los estilos de gestión educativa y el Clima Institucional en IE Públicas del distrito de Carmen de la Legua.

\section{Específicos}

- Establecer la correlación que hay entre el estilo autoritario y el Clima Institucional de IE Públicas del distrito de Carmen de la Legua.

- Establecer la relación que hay entre el estilo de gestión democrático y el Clima Institucional en IE Públicas del distrito de Carmen de la Legua.

- Establecer la relación que hay entre el estilo de gestión Leissez Faire y el Clima institucional en IE Públicas del distrito de Carmen de la Legua.

\section{MÉTODOS}

\section{Tipo de estudio}

El presente trabajo de investigación es de carácter no experimental - tipo básico.

\section{Diseño}

El presente estudio aplicó un diseño, el cual es el descriptivo Correlacional. El estudio nos explica la relación o asociación no causal que hay entre las variables. En primer lugar se miden las variables y después a través de pruebas de hipótesis correlaciónales y la consiguiente aplicación de técnicas estadísticas se determina la correlación.

Si bien la investigación correlacional no establece de forma directa relaciones causales, puede dar indicios sobre las posibles causas de un fenómeno (Mejía, 2011).

\section{Procedimiento y Técnica}

La técnica a utilizar es la encuesta en la cual los dos instrumentos están basados.

\section{Tipo de Prueba de Hipótesis}

Los datos obtenidos a partir del proceso de observación fueron ordenados y trasladados hacia 
una data estadística. Luego se utilizó el software estadístico SPSS v 24 para la aplicación de los estadígrafos correspondientes al tipo de estudio. Luego de obtenidos los gráficos y cuadros se procedió a su interpretación.

\section{Prueba de Hipótesis: Rho Spearman}

Para el proceso de prueba de hipótesis, en el que se verifica la diferencia de medias, se tomó en cuenta el siguiente procedimiento:

$$
\begin{aligned}
& p=1-\frac{6 \Sigma d^{2}}{n\left(n^{2}-1\right)} \\
& r_{s}=1-\frac{6 \Sigma d^{2}}{n\left(n^{2}-1\right)}
\end{aligned}
$$

$r_{s}=$ Coeficiente de correlación por rangos de Spearman

$\mathrm{d}=$ Diferencia entre los rangos (X menos $\mathrm{Y}$ )

$\mathrm{n}=$ Número de datos

\section{Prueba de Kolmogórov Smirnov}

Para determinar la normalidad de los datos, es decir si cada una de las dos poblaciones que están siendo comparadas sigue una distribución normal.

\section{Modelo estadístico de la prueba Chi cuadrado}

Se utiliza la Prueba Chi cuadrado teniendo como base la muestra, las hipótesis y los supuestos. Los datos son no paramétricos de tipo ordinal.

Se utilizó la siguiente tabla de comparación para establecer el nivel de correlación:

\section{Población y Muestra}

El universo para el presente trabajo de investigación estuvo constituido por los docentes de dos instituciones educativas de gestión pública de nivel secundaria que están dentro de la jurisdicción del distrito de Carmen de la Legua. La muestra fue censal pues se utilizó toda la muestra de 67 docentes.

\section{Instrumento de la Investigación}

Los instrumentos que se utilizaron fueron dos: cuestionario, para medir el nivel de las variables: Clima Institucional y el otro instrumento: Lista de cotejo, para calificar a la variable estilo de gestión.

Encontramos que la confiabilidad del instrumento de estilo de gestión es de 0,67 la cual según el cuadro de Confiabilidad de Kuder Richardson es muy confiable al encontrarse en el rango de 0,66 a 0,71 y según el análisis estadístico Alfa de Cron Bach nuestro instrumento de clima Institucional tiene alta confiabilidad al tener $\alpha=0,775$.

En total se ha tenido dos instrumentos, en la siguiente distribución:

Tabla 1.

Distribución de los instrumentos

\begin{tabular}{lccc} 
VARIABLE & Cuestionario & Lista de cotejo & TOTAL \\
\hline Clima institucional & Docentes & $\mathrm{X}$ & 1 \\
Estilo de Gestión & $\mathrm{X}$ & Docentes & 1 \\
Total & & & 2 \\
\hline
\end{tabular}

\section{RESULTADOS: Análisis y discusión}

Los datos obtenidos a partir del proceso de observación fueron ordenados y trasladados hacia una data estadística. Luego se utilizó el

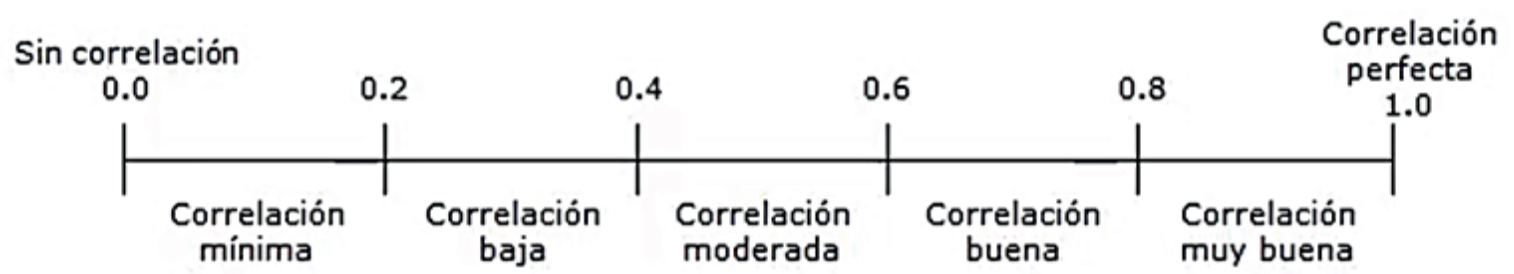


software estadístico SPSS para la aplicación de los estadígrafos correspondientes al tipo de estudio.

Luego de obtenidos los gráficos y cuadros se procedió a su interpretación.

Encontramos en la Tabla 2, que 3 docentes que consideran que la gestión es de estilo autoritario que equivale el $14,3 \%$ consideran que el Clima Institucional es bajo, por otro lado 16 docentes que equivalen a un $76,2 \%$ considera que el Clima es regular y 2 docentes es decir el 9.5\% considera que el Clima es muy bueno. En la figura 1 podemos observar que en el estilo de gestión autoritario el clima institucional que impera es el regular con un $76,2 \%$.

Encontramos en la Tabla 3, que 2 docentes que consideran que la gestión es de estilo democrático que equivale el $8 \%$ consideran que el Clima Institucional es bajo, por otro lado 2 docentes que equivalen a un $8 \%$ consideran que el Clima institucional es regular y 21 docentes es decir el $84 \%$ considera que el clima institucional es Muy Bueno. En la fig. 2 podemos observar que en el estilo de gestión democráti-

Tabla 2.

Estilo de Gestión: AUTORITARIO

\begin{tabular}{llcccc}
\multicolumn{2}{c}{} & \multicolumn{2}{c}{ Clima Institucional } \\
& & Frecuencia & Porcentaje & Porcentaje válido & $\begin{array}{c}\text { Porcentaje acumu- } \\
\text { lado }\end{array}$ \\
\multirow{3}{*}{ Válidos } & Bajo & 3 & 14,3 & 14,3 & 14,3 \\
& Regular & 16 & 76,2 & 76,2 & 90,5 \\
& Muy Bueno & 2 & 9,5 & 9,5 & 100,0 \\
\hline
\end{tabular}

a. Estilo de Gestión = Autoritario

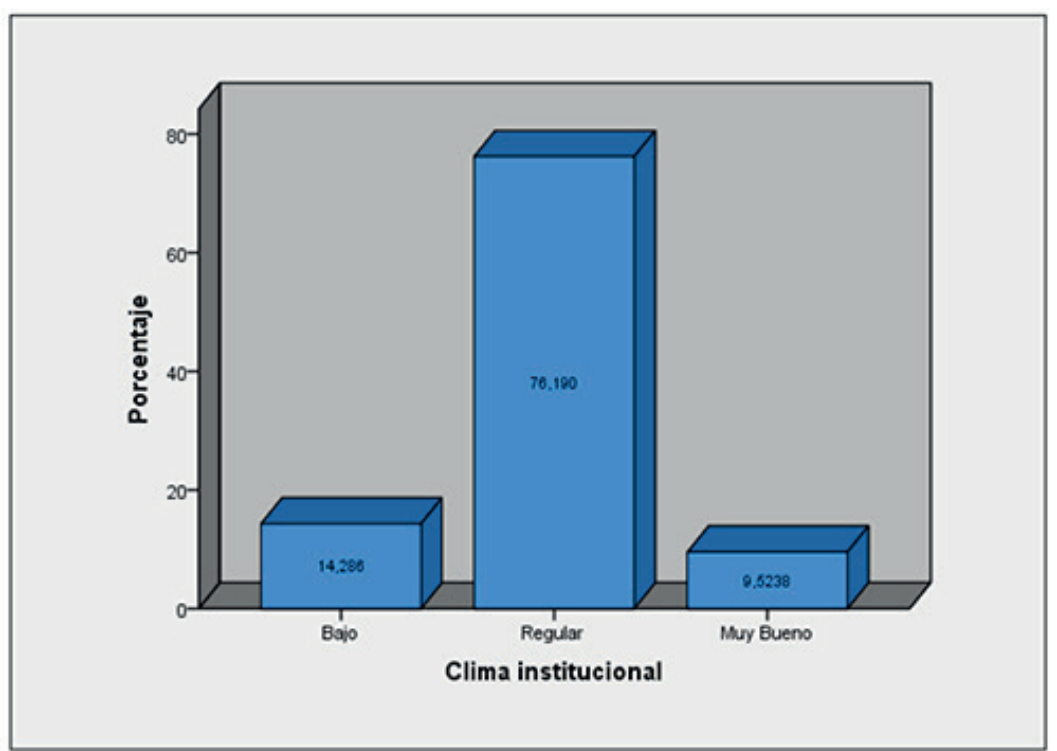

Figura 1. Estilo de gestión Autoritario

Tabla 3.

Estilo de Gestión: DEMOCRÁTICO

\begin{tabular}{llcccc} 
& & \multicolumn{2}{c}{ Clima Institucional $^{\mathbf{a}}$} & & \\
& & Frecuencia & Porcentaje & Porcentaje válido & Porcentaje acumulado \\
\multirow{2}{*}{ Válidos } & Bajo & 2 & 8,0 & 8,0 & 8,0 \\
& Regular & 2 & 8,0 & 8,0 & 16,0 \\
& Muy Bueno & 21 & 84,0 & 84,0 & 100,0 \\
\hline
\end{tabular}

a. Estilo de Gestión = democrático 


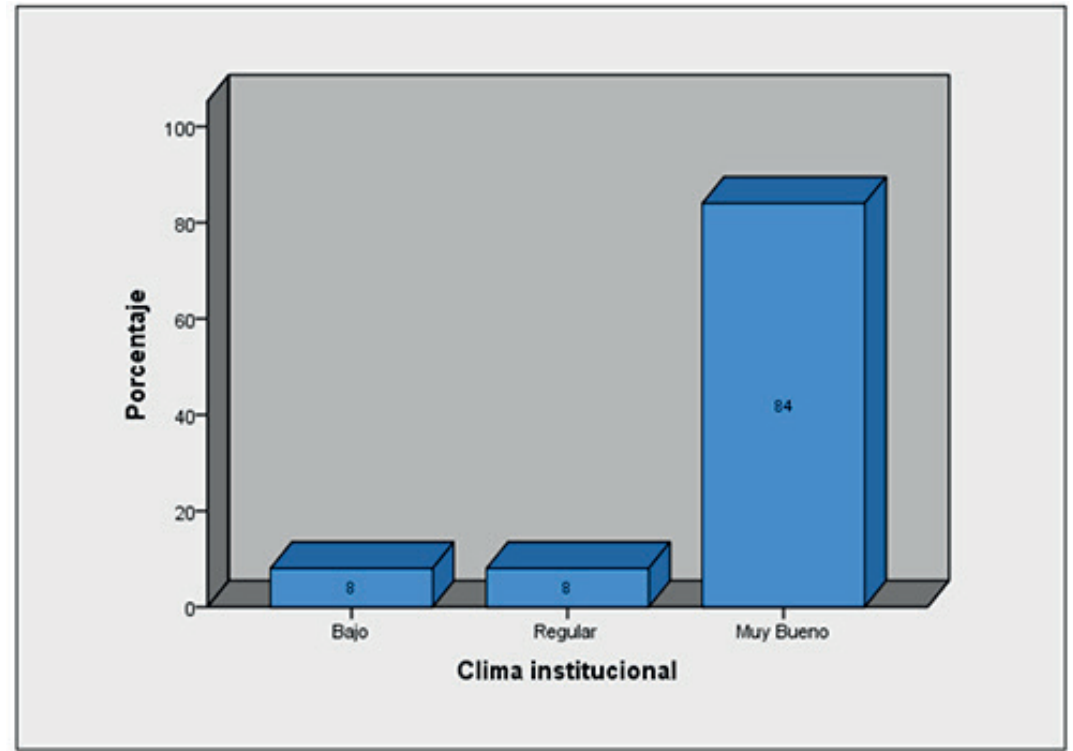

Figura 2. Estilo de gestión democrático

co el Clima institucional que impera es el Muy Bueno con un $84 \%$.

Encontramos en la Tabla 4, que 3 docentes que consideran que la gestión es de estilo LEISSEZ que equivale el $14,3 \%$ consideran que el cli- ma es Bajo, por otro lado 4 docentes que equivalen a un $19 \%$ considera que el clima es regular y 14 docentes es decir el $66.7 \%$ considera que el clima es Bueno. En la figura 3 podemos observar que en el estilo de gestión Leissez el clima institucional que impera es el Bueno con un 66.7\%.

Tabla 4.

Estilo de gestión. Leissez

\begin{tabular}{clcccc}
\multicolumn{5}{c}{} & \multicolumn{3}{c}{ Clima Institucional $^{\mathbf{a}}$} \\
\multirow{3}{*}{ Válidos } & Frecuencia & Porcentaje & Porcentaje válido & Porcentaje acumulado \\
& Bajo & 3 & 14,3 & 14,3 & 14,3 \\
& Regular & 4 & 19,0 & 19,0 & 33,3 \\
& Bueno & 14 & 66,7 & 66,7 & 100,0
\end{tabular}

a. Estilo de Gestión = Leissez

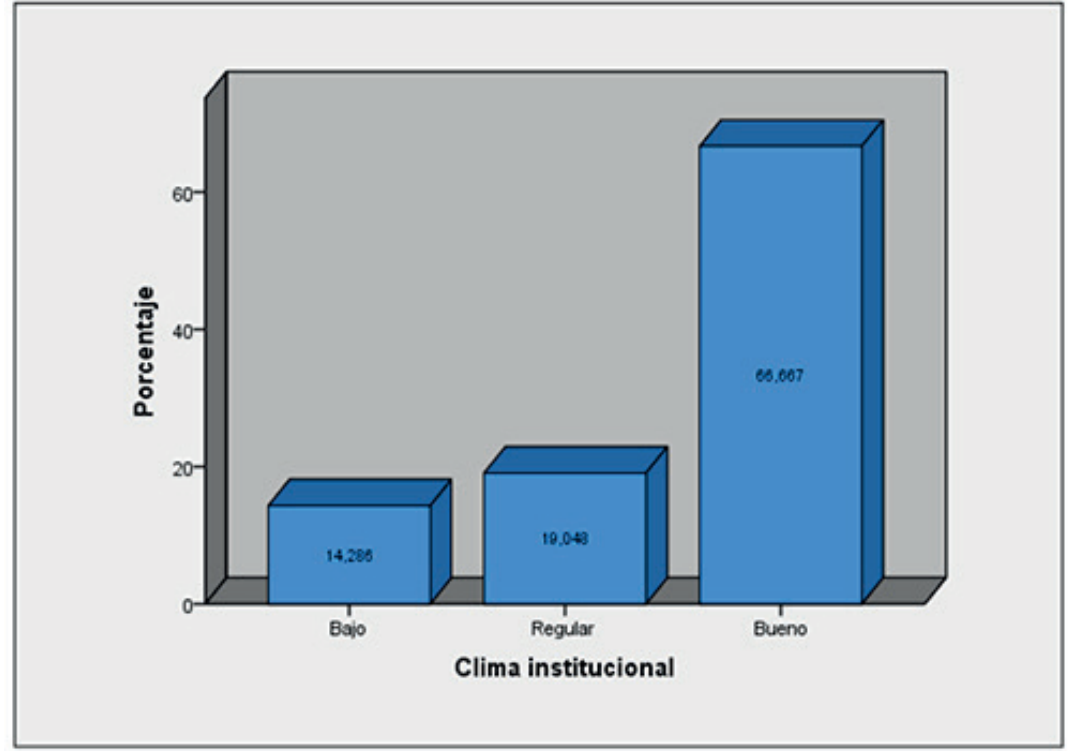

Figura 3. Estilo de Gestión: LEISSEZ 
Estadística Inferencial

Hipótesis General

$\mathrm{H}_{1}$ : Existe relación significativa entre los estilos de gestión educativa y el clima institucional de IE públicas en el distrito Carmen de La Legua.

$\mathrm{H}_{0}$ : No existe relación significativa entre los estilos de gestión educativa y el clima institucional de IE Públicas en el distrito de Carmen de La Legua.
Según nuestra tabla de contingencia encontramos que tiene una asociación bilateral

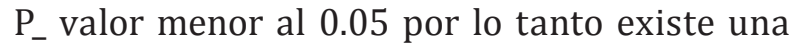
asociación significativa al 95\% rechazando la hipótesis nula y aceptando la alterna.

\section{Hipótesis secundaria 1}

$\mathrm{H}_{1}$ : Existe relación significativa entre el estilo autoritario y el clima institucional de IE Públicas del distrito de Carmen de La legua.

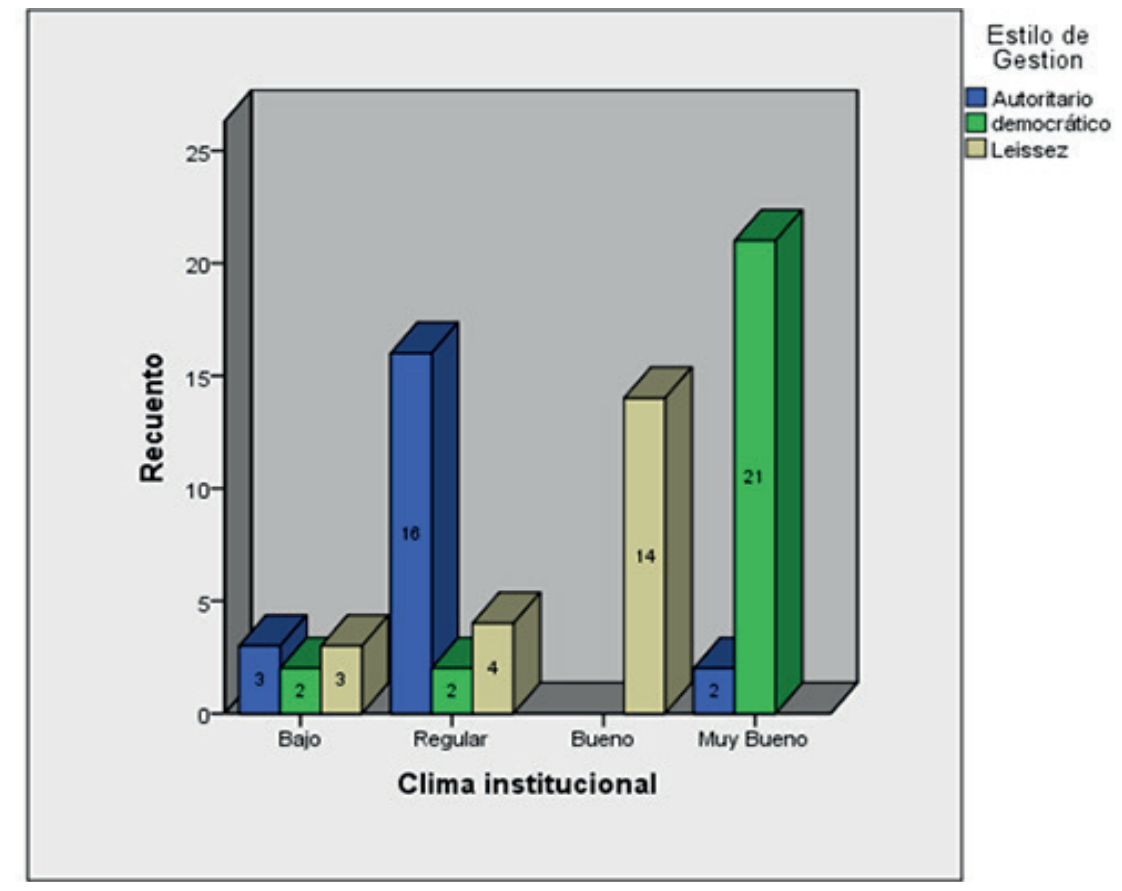

Figura 4. Barras de Clima institucional según estilo de gestión

Tabla 5.

De contingencia Clima institucional * Estilo de Gestión

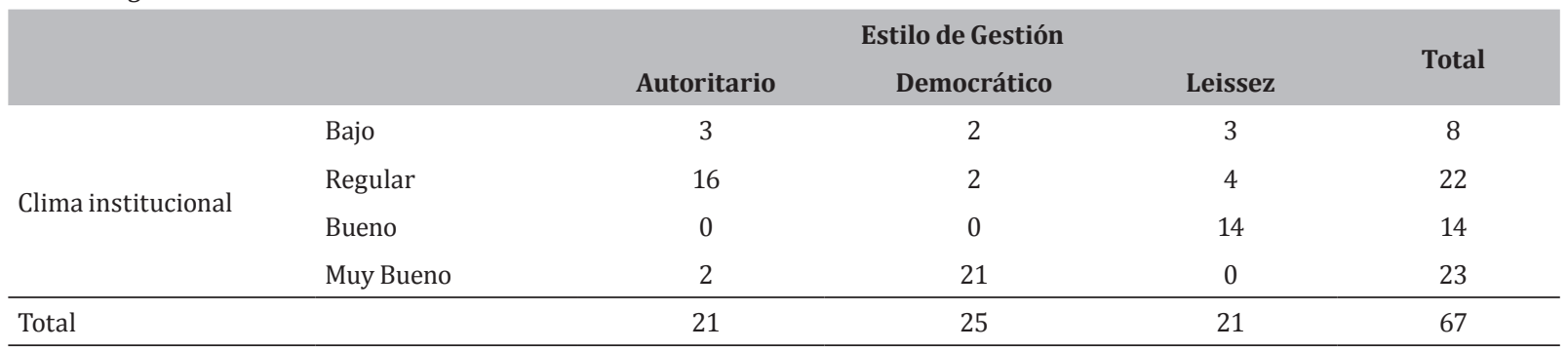

Pruebas de Chi cuadrado

\begin{tabular}{lccc} 
& valor & gl & Sig. Asintótica (bilateral) \\
Chi cuadrado de Pearson & $78,059^{\mathrm{a}}$ & 6 &, 000 \\
Razón de verosimilitudes & 82,420 & 6 &, 000 \\
Asociación lineal por lineal & 2,134 & 1 &, 144 \\
$\mathrm{~N}^{\circ}$ de casos válidos & 67 & & \\
\hline
\end{tabular}

a. 0 casillas $(0 \%)$ tienen una frecuencia esperada inferior a 5 .

La frecuencia mínima esperada es 2,51 
$\mathrm{H}_{0:}$ No existe relación significativa entre el estilo autoritario y el clima institucional de IE públicas del distrito de Carmen de la Legua.

Pruebas de Chi cuadrado

\begin{tabular}{lccc} 
& valor & gl & $\begin{array}{c}\text { Sig. Asintótica } \\
\text { (bilateral) }\end{array}$ \\
Chi cuadrado de Pearson & $78,059^{a}$ & 6 &, 000 \\
Razón de verosimilitudes & 82,420 & 6 &, 000 \\
Asociación lineal por lineal & 2,134 & 1 &, 144 \\
$\mathrm{~N}^{\circ}$ de casos válidos & 67 & & \\
\hline
\end{tabular}

a. 0 casillas $(0 \%)$ tienen una frecuencia esperada inferior a 5 . La frecuencia mínima esperada es 2,51

Según nuestra tabla de contingencia encontramos que tiene una asociación bilateral p_ valor menor al 0.05 por lo tanto existe una asociación significativa al 95\% rechazando la hipótesis nula y aceptando la alterna:

Existe relación significativa entre el estilo autoritario de los directivos y el clima institucional de EI Públicas en el distrito de Carmen de la Legua.

\section{Hipótesis secundaria 2}

$\mathrm{H}_{1}$ : Existe relación significativa entre el estilo democrático de los directivos y el clima institucional de IE Públicas del distrito de Carmen de la Legua.

$\mathrm{H}_{0}$ : No existe relación significativa entre el estilo democrático de los directivos y el clima institucional de IE Públicas del distrito de Carmen de la Legua.

Pruebas de Chi cuadrado

\begin{tabular}{lccc} 
& valor & gl & $\begin{array}{c}\text { Sig. Asintótica } \\
\text { (bilateral) }\end{array}$ \\
Chi cuadrado de Pearson & $78,059^{\mathrm{a}}$ & 6 &, 000 \\
Razón de verosimilitudes & 82,420 & 6 &, 000 \\
Asociación lineal por lineal & 2,134 & 1 &, 144 \\
$\mathrm{~N}^{\circ}$ de casos válidos & 67 & & \\
\hline
\end{tabular}

a. 0 casillas (0\%) tienen una frecuencia esperada inferior a 5 . La frecuencia mínima esperada es 2,51

Según nuestra tabla de contingencia encontramos que tiene una asociación bilateral p_ valor menor al 0.05 por lo tanto existe una asociación significativa al 95\% rechazando la hipótesis nula y aceptando la alterna:
Existe relación significativa entre el estilo democrático de los directivos y el clima institucional de IE Públicas en el distrito Carmen de la Legua.

\section{Hipótesis secundaria 3}

$\mathrm{H}_{1}$ : Existe relación significativa entre el estilo Leissez Faire de los directivos y el clima institucional de IE Públicas en el distrito Carme de la Legua.

$\mathrm{H}_{0}$ : No existe relación significativa entre el estilo Leissez Faire de los directivos y el clima institucional de IE Públicas del distrito de Carme de la Legua.

\section{Pruebas de Chi cuadrado}

\begin{tabular}{lccc} 
& Valor & gl & $\begin{array}{c}\text { Sig. asintótica } \\
\text { (bilateral) }\end{array}$ \\
Chi-cuadrado de Pearson & $78,059^{\mathrm{a}}$ & 6 &, 000 \\
Razón de verosimilitudes & 82,420 & 6 &, 000 \\
Asociación lineal por lineal & 2,134 & 1 &, 144 \\
N de casos válidos & 67 & & \\
\hline
\end{tabular}

a. 0 casillas $(0 \%)$ tienen una frecuencia esperada inferior a 5 . La frecuencia mínima esperada es 2,51.

Según nuestra tabla de contingencia encontramos que tiene una asociación bilateral p_ valor menor al 0.05 por lo tanto existe una asociación significativa al 95\% rechazando la hipótesis nula y aceptando la alterna:

Se evidencia que hay una correlación significativa entre el estilo Leissez Faire y el clima institucional en IE Públicas del distrito Carmen de la Legua.

\section{CONCLUSIONES}

- Se concluye que existe una correlación significativa entre los estilos de gestión educativa y el Clima institucional en IE Públicas del distrito de Carmen de la legua.

- Se concluye que al imperar el estilo de gestión democrático el clima institucional es Muy Bueno en IE Públicas del distrito de Carmen de la Legua.

- Se concluye que al imperar el estilo de gestión Leissez Faire el clima institucional es Bueno en IE Públicas del distrito de Carmen de la Legua. 


\section{REFERENCIAS BIBLIOGRÁFICAS}

Alvarado 0. (2000). Gerencia educativa. Desafíos y oportunidades. Ediciones Vallejianas. Lima.

Arana, María (1998) Principios y Procesos de la gestión educativa. Editorial San Marcos. Lima - Perú.

Collao, Oscar. (1998) Administración y Gestión Educativa. Editorial San Marcos, Lima - Perú.

Gamarra, Isabel (2011) Estilos de gestión de los directivos y su relación con la calidad de la con- ducción de las instituciones educativas de ex variante técnica de la UGEL 03 de Lima. Tesis para optar el grado académico de Maestría en gestión de la educación. UNMSM.

Hernández Sampieri, Roberto (2003) Metodología de la Investigación. Mac Graw Hill Interamericana S.A Colombia.

Mejía, Elías (2011) Metodología de la investigación Científica UNMSM. Lima - Perú.

Pineda, Elia et al (1994) Metodología de la investigación OPS. OMS 2da Edición. 